\title{
THE PLASTIC COLLAPSE OF CYLINDRICAL SHELLS UNDER AXIALLY SYMMETRICAL LOADING*
}

\author{
BY \\ E. T. ONAT \\ Brown University
}

1. Introduction. This paper is concerned with the plastic behavior and the load carrying capacities of thin cylindrical shells composed of a plastic-rigid material that obeys Tresca's yield condition and the associated flow rule. The discussion is restricted to axially symmetric types of loading and edge support. Under these conditions a yield surface is found for the relevant stress resultants. The method employed in the determination of the yield surface could be extended to the case of general loading of the shells of arbitrary shape.

Once the yield surface and the associated flow rule are known, the approximations from above and below to the load carrying capacity can be obtained by using the theorems of limit analysis $[1,2,3]^{* *}$. For mathematical simplification it is found convenient to approximate the curved yield surface by a polyhedron or other suitable shapes. A simple example is treated using this approximate approach.

2. Notations. Equations of equilibrium. Velocity strains. Let $r, \varphi, x$ be cylindrical coordinates, the $x$ axis being horizontal, and let the considered shell be bounded by the cylinders $r=R \pm h / 2$, where $R$ is the radius of the middle surface. An element is cut from the shell by two adjacent planes through the $x$ axis and two adjacent planes perpendicular to the $x$ axis. The stress resultants acting on the sides of the element are shown in Fig. 1.

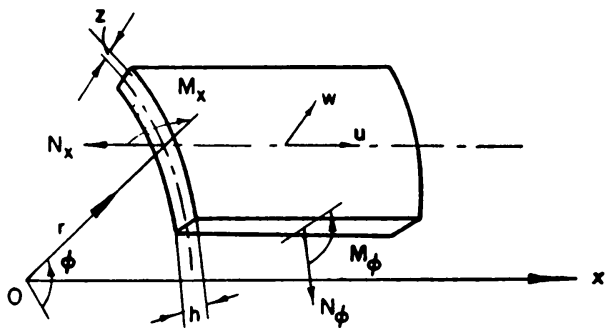

Frg. 1

On account of the axial symmetry of loading and support, the membrane shearing forces $N_{x \varphi}=N_{\varphi x}$ and the twisting moments $M_{x \varphi}=M_{\varphi x}$ vanish identically and the bending moments $M_{\varphi}$ are constant along the circumference.

As regards the transverse shearing forces, it follows from the assumed rotational symmetry that only the forces $Q_{x}$ are different from zero. Furthermore, if the shell thickness $h$ is small compared with the radius $R$, then, the stresses $\sigma_{r}$ and $\tau_{r x}$ may be regarded as small when compared with the stresses $\sigma_{z}$ and $\sigma_{\varphi}$. The state of stress at a

${ }^{*}$ Received April 12, 1954. The results presented in this paper were obtained in the course of research conducted under Contract DA-19-020-0RD-798.

**The numbers in the square brackets refer to the Bibliography at the end of the paper. 
generic point of the shell is essentially two-dimensional and on account of the rotational symmetry $\sigma_{z}$ and $\sigma_{\varphi}$ are the relevant principal stresses.

These stresses give rise to the principal bending moments

$$
M_{z}=-\int_{-h / 2}^{+h / 2} \sigma_{z} z d z, \quad M_{\bullet}=-\int_{-h / 2}^{+\Lambda / 2} \sigma_{\varphi} z d z
$$

and to the principal membrane forces

$$
N_{x}=\int_{-h / 2}^{+h / 2} \sigma_{x} d z, \quad N_{\varphi}=\int_{-k / 2}^{+h / 2} \sigma_{\varphi} d z .
$$

The shearing stress $\tau_{r z}$ gives rise to the shear force

$$
Q_{x}=-\int_{-h / 2}^{+h / 2} \tau_{r x} d z
$$

If the three-dimensional conditions of equilibrium for local stresses are replaced by two-dimensional conditions of equilibrium for the stress resultants, the following equations are obtained.

$$
\begin{gathered}
\frac{d N_{x}}{d x}=0 \text { (or equal to the body force in } x \text { direction), } \\
\frac{d Q_{x}}{d x}+\frac{N_{Q}}{R}=p, \quad \frac{d M_{x}}{d x}-Q_{x}=0,
\end{gathered}
$$

where $p$ is the distributed external load acting in the positive $r$ direction. These equations are independent of the mechanical properties of the shell material.

As regards the incipient plastic flow, following the general theory of thin shells we assume that the velocity field is such that the normals of the undeformed middle surface remain normal to the deformed middle surface. Since we are concerned with the axially symmetrical deformations of the shell, the component $v$ of the velocity in the circumferential direction is zero, and we have to consider only the components $u$ and $w$ in the $x$ and $r$ directions.

The expressions for the strain rates at a generic point of the shell situated at a distance $z$ from the middle surface are then given by the following equations:

$$
e_{x}=\epsilon_{x}-z \kappa, \quad e_{\varphi}=\epsilon_{\varphi}, \quad e_{r \varphi}=e_{r x}=e_{x \varphi}=0,
$$

where

$$
\epsilon x=\frac{\partial u}{\partial x}, \quad \epsilon_{\varphi}=\frac{w}{R}
$$

are the principal extension rates of the middle surface and

$$
\kappa=\frac{\partial^{2} w}{\partial x^{2}}
$$

is the rate of curvature change of the middle surface in the $x$ direction.

The radial rate of strain follows from the incompressibility of the shell material:

$$
e_{r}=-\left(e_{p}+e_{x}\right) \text {. }
$$


3. Plastic behavior of a typical element. Yield condition for stress resultants. The perfectly plastic material considered in this paper is supposed to obey Tresca's yield condition. For the case of plane stress which is a good approximation to the present problem this yield condition is indicated in Fig. 2. All points on the yield hexagon in

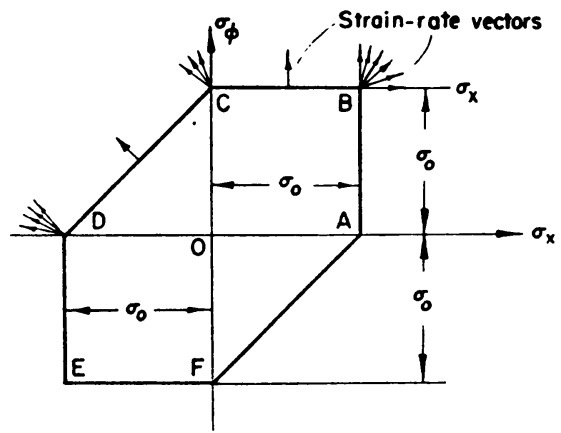

Fig. 2

this figure represent states of stress for which the maximum shearing stress has the constant value of $\sigma_{0} / 2$.

It is convenient to speak of the set of plastic states represented by interior points of the segment $A B$ as the plastic regime $A B$, etc.; similarly, the plastic state represented by the vertex $A$ of the yield hexagon will be the plastic regime $A$, etc.

The flow rule associated with this yield condition states that the strain-rate vector which has the principal strain-rates $e_{x}$ and $e_{\varphi}$ as components is perpendicular to the sides of the yield hexagon for states of stress represented by points other than the vertices of the hexagon. At the vertices the strain-rate vector is obtained by the linear combination (with positive coefficients) of the strain-rate vectors corresponding to the adjacent sides of the hexagon.

The third principal strain is found from the equation of incompressibility (8).

Now if the element considered in Fig. 1 is to bend plastically, the yield condition and the associated flow rule must be fulfilled in each layer parallel to the middle surface.

On the other hand Eqs. (5) show that the strain-rate vector in a layer is a function of $z$ (indicating the position of the layer) and three parameters-the principal strain rates $\epsilon_{x}, \epsilon_{\varphi}$ and the rate of change of curvature of middle surface $\kappa$. Since the magnitude of the strain-rate vector is of no importance in determining the corresponding state of stress, there are essentially two parameters; hence $\epsilon_{x} / \kappa, \epsilon_{\varphi} / \kappa$ can be chosen as characteristic parameters. From the flow rule stated above we conclude that the stresses $\sigma_{x}$ and $\sigma_{\varphi}$ are also functions of $z$ and $\epsilon_{x} / \kappa, \epsilon_{\varphi} / \kappa$.

If we then compute the stress-resultants as defined by Eqs. (1) and (2), we see that the stress-resultants $N_{x}, N_{\varphi}, M_{x}$ and $M_{\Phi}$ corresponding to the plastic state of the shell element are functions of the two parameters $\epsilon_{x} / \kappa$ and $\epsilon_{\varphi} / \kappa$. Therefore if the stress resultants $N_{x}, N_{\varphi}$ and $M_{x}$ (which occur in the equilibrium equations) are used as rectangular coordinates, the plastic states of the shell element define a surface. This surface will be called the yield surface for the stress resultants $N_{x}, N_{\varphi}$ and $M_{x}$. Leaving to the next section the formulation of a boundary value problem with the help of this surface and of the equations of equilibrium, we proceed to find the exact shape of the yield surface. 
Consider $\epsilon_{x} / \kappa$ and $\epsilon_{\varphi} / \kappa$ as rectangular coordinates and assume that $\kappa>0$. The vector OP with the components $\epsilon_{a} / \alpha$ and $\epsilon_{p} / \kappa$ is the strain-rate vector of the middle surface. If on the other hand a vector $O Q$ is constructed as in Fig. 3 , it is easily found from the

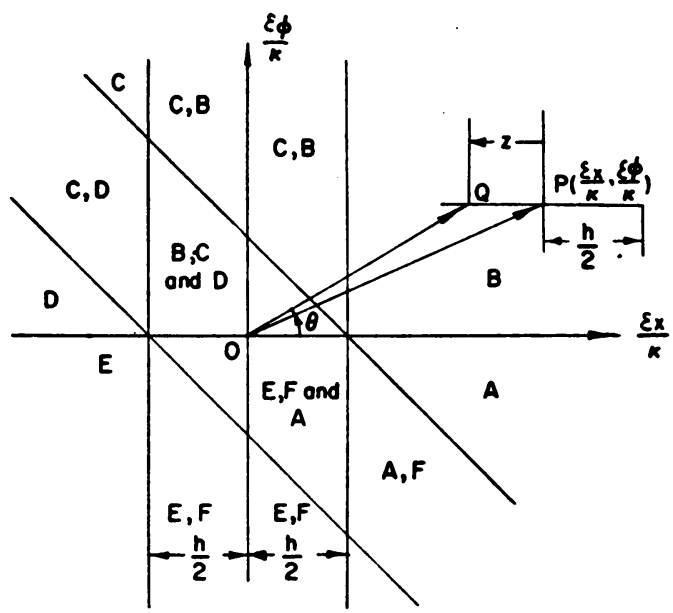

Fig. 3

Eqs. (5) that $O Q$ represents the strain-rate vector in the layer situated at a distance $z$ from the middle surface of the shell. It is seen from Fig. 3, that

$$
\frac{e_{z}}{e_{p}}=\left(\frac{\epsilon_{z}}{x}-z\right) / \frac{\epsilon_{p}}{x}=\operatorname{ctg} \theta,
$$

where $\theta$ is the angle of inclination of the strain-rate vector.

Once $\theta$ is determined, the corresponding stress state is found from the flow rule; for example, in the case considered, $0<\theta<\pi / 2$, therefore the stress-state corresponding to $O P$ (and $O Q$ ) is the regime $B$. Moreover if $P$ is situated in the domain defined by the inequalities

$$
\frac{\epsilon_{x}}{\kappa}>\frac{h}{2}, \quad \frac{\epsilon_{p}}{\kappa}>0
$$

a similar investigation of strain-rate vectors indicates that every layer of the sheet is in the regime $B$. Similar regions are determined and shown in Fig. 3, the capital letters denoting the various plastic regimes existing across the thickness of the shell; for instance if $P$ is in the region denoted by $C, B$, the layers of the shell are either in the plastic regime $C$ or $B$.

By considering the stress-states corresponding to the domains indicated in Fig. 3 one can easily obtain the complete yield surface of stress resultants. Previous remarks indicate that the region $B$ of Fig. 3 is mapped to one point $\left(B^{\prime}\right)$ of the yield surface, where

$$
N_{\varphi}=N_{x}=\sigma_{0} h \quad \text { and } \quad M_{x}=0 .
$$

Since $B$ corresponds to a two-parameter family of strain-rate vectors, we must expect $B^{\prime}$ to be a corner of the yield surface. Similar considerations apply to the domains $C, D, E, A$.

Inspection shows that interesting information is obtained if the parellelogram shaped region in Fig. 3 is considered. In Fig. 4 the part of this region where $\epsilon_{\varphi} / \kappa>0$ is indicated. 
If a generic point $P$ representing the strain in the middle surface is considered the previous remarks enable us to determine the stress distribution on the element shown in Fig. 1:

$$
\begin{aligned}
& \sigma_{x}=-\sigma_{0} \quad \text { when } \quad \frac{\epsilon_{\varphi}}{\kappa}+\frac{\epsilon_{x}}{\kappa}<z<\frac{h}{2}, \\
& \sigma_{x}=0 \quad \text { when } \quad \frac{\epsilon_{x}}{\kappa}<z<\frac{\epsilon_{\varphi}}{\kappa}+\frac{\epsilon_{x}}{\kappa} \\
& \sigma_{x}=\sigma_{0} \quad \text { when } \quad-\frac{h}{2}<z<\frac{\epsilon_{x}}{\kappa}, \\
& \sigma_{\varphi}=0 \quad \text { when } \quad \frac{\epsilon_{\varphi}}{\kappa}+\frac{\epsilon_{x}}{\kappa}<z<\frac{h}{2}, \\
& \sigma_{\varphi}=\sigma_{0} \quad \text { when } \quad-\frac{h}{2}<z<\frac{\epsilon_{\varphi}}{\kappa}+\frac{\epsilon_{x}}{\kappa}, \\
& \sigma_{0}
\end{aligned}
$$

FIG. 4

where the condition that $P$ should be in the region considered is expressed by the following inequalities

$$
0<\frac{\epsilon_{\varphi}}{\kappa}<-\frac{\epsilon_{x}}{\kappa}+\frac{h}{2}, \quad-\frac{h}{2}<\frac{\epsilon_{x}}{\kappa}<+\frac{h}{2} .
$$

From (1), (2) and (9) we can obtain the corresponding stress resultants as functions of $\epsilon_{z} / k$ and $\epsilon_{v} ; k$ :

$$
\left.\begin{array}{c}
\frac{N_{x}}{\sigma_{0} h}=\frac{1}{2}\left(\frac{\epsilon_{\varphi}}{\kappa}+2 \frac{\epsilon_{x}}{\kappa}\right), \quad \frac{N_{\varphi}}{\sigma_{0} h}=\frac{1}{2}+\frac{\epsilon_{\varphi}}{h \kappa}+\frac{\epsilon_{x}}{h \kappa^{\prime}} \\
\frac{M_{x}}{\sigma_{0} h^{2} / 4}=1-\frac{2}{h^{2}}\left[\left(\frac{\epsilon_{x}}{\kappa}\right)^{2}+\left(\frac{\epsilon_{\varphi}}{\kappa}+\frac{\epsilon_{x}}{\kappa}\right)^{2}\right] .
\end{array}\right\}
$$


Equations (11), together with the inequalities (10) define a bounded surface in $N_{x}, N_{\varphi}$ and $M_{x}$ space.

If we put $N_{0}=\sigma_{0} h, M_{0}=\sigma_{0} h^{2} / 4$, and eliminate $\epsilon_{x} / \kappa$ and $\epsilon_{\varphi} / \kappa$ from (11), then we obtain:

$$
F\left(\frac{N_{x}}{N_{0}}, \frac{N_{\varphi}}{N_{0}}, \frac{M_{x}}{M_{0}}\right)=\frac{M_{x}}{M_{0}}-1+2\left\{\left[\frac{N_{x}}{N_{0}}-\left(\frac{N_{\varphi}}{N_{0}}-\frac{1}{2}\right)\right]^{2}+\left(\frac{N_{\varphi}}{N_{0}}-\frac{1}{2}\right)^{2}\right\}=0 .
$$

Moreover the inequalities (10) can be written in terms of stress resultants

$$
\frac{1}{2} \frac{N_{x}}{N_{0}}+\frac{1}{2}<\frac{N_{\varphi}}{N_{0}}<1, \quad 1<\frac{N_{x}}{N_{0}}-\frac{N_{\varphi}}{N_{0}}<0 .
$$

The surface defined by (12) and (13) is bounded by the parabolas $B^{\prime} \beta C^{\prime}, C^{\prime} \alpha D^{\prime}$ and $D^{\prime} \gamma B^{\prime}$ (Fig. 6). Three different projections of this surface are also indicated in Figs. $5 \mathrm{a}, 5 \mathrm{~b}$ and $5 \mathrm{c}$. It can be shown that by considering the other half of the parallelogram

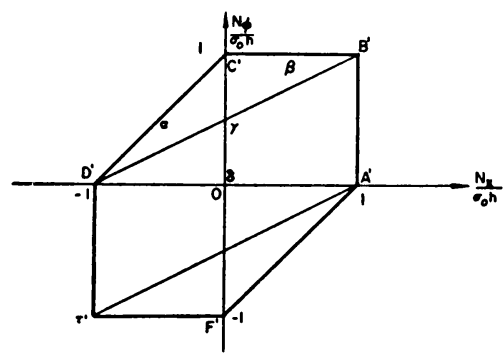

(e)

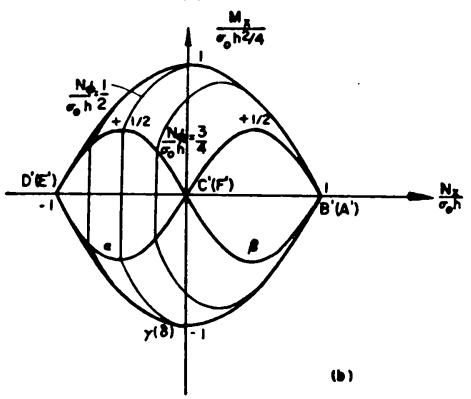

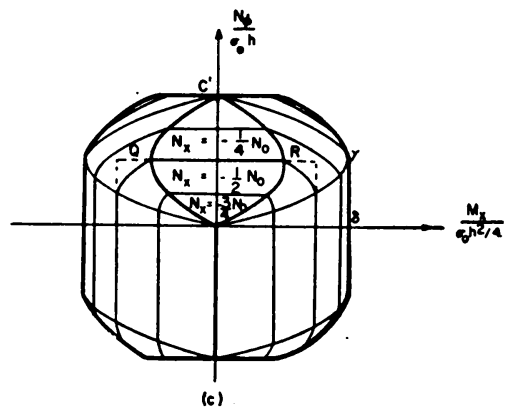

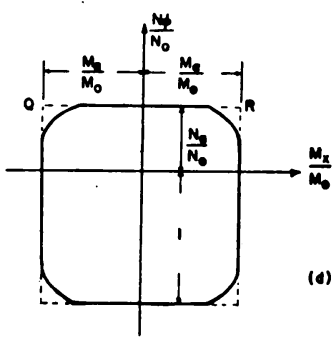

FIG. 5

and also by investigating the case $\kappa<0$ one obtains three bounded surfaces which are identical with the surface defined by (12) and (13). These surfaces are symmetrically situated with respect to $M_{x} / M_{0}=0$ plane.

The study of the particular case $\kappa=0$ adds four flat pieces bounded by the parabolic $\operatorname{arcs} B^{\prime} \beta C^{\prime}$ and $C^{\prime} \alpha D^{\prime}$ and their symmetrical counterparts. Moreover the investigation of the case $\epsilon_{\varphi}=0$ gives two right parabolic cylinders whose bases $B^{\prime} \gamma D$ and its symmetrical counterpart are shown in Fig. 5b. These cylinders are tangential to the surfaces of the type defined by the Eqs. (12) and (13). With this investigation the determination of the yield surface is completed and the resulting convex surface is shown in Figs. [5(a, b, c)] and Fig. 6 .

It should be noted that the yield curves obtained by the intersection of the planes 
$N_{x}=0$ and $N_{\varphi}=0$ with this yield surface were previously investigated respectively by Drucker [4] and Onat and Prager [5].

Points within the domain bounded by the yield surface of Fig. 6 represent "safe" combinations of axial forces and bending moment, while points on this surface represent critical combinations of the stress resultants.

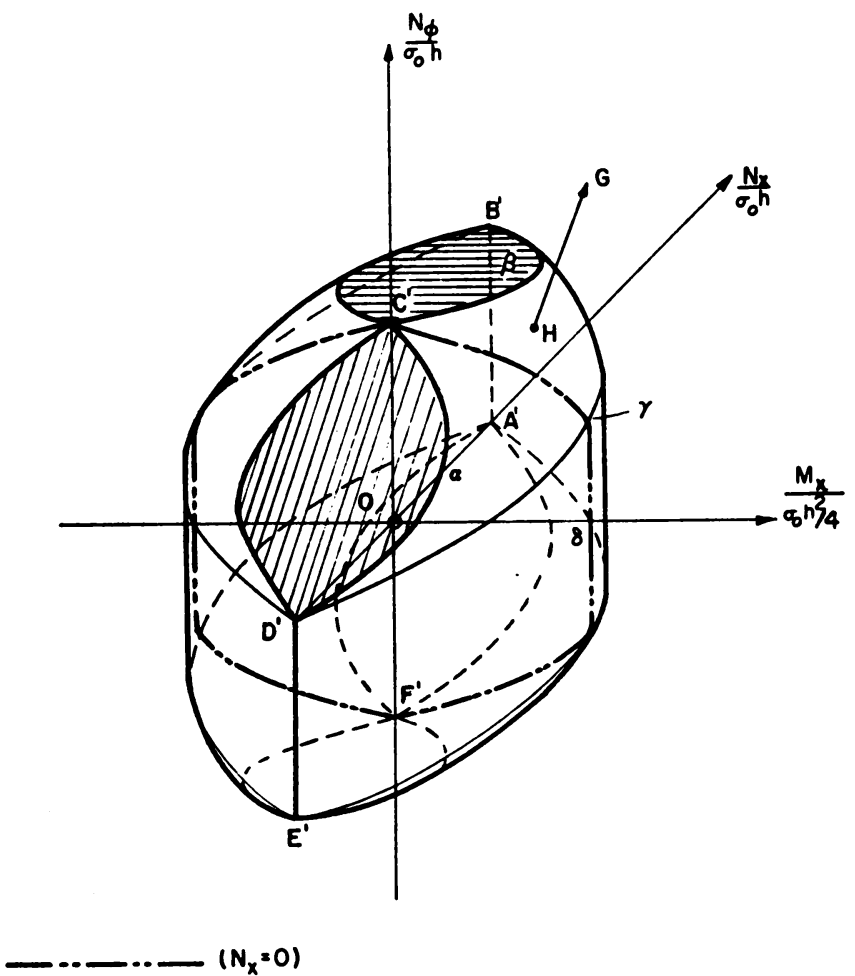

FIG. 6

Under any such critical combination of $N_{x}, N_{\varphi}$ and $M_{\varphi}$ a certain type of deformation becomes possible which is characterized by the ratios between $\epsilon_{x}, \epsilon_{\varphi}$ and $\kappa$.

If the critical combination is represented by a point $\left(N_{x} / N_{0}, N_{\varphi} / N_{0}, M_{x} / M_{0}\right)$ on the surface defined by Eq. (12), then the corresponding flow mechanism $\left(\epsilon_{x} / \kappa, \epsilon_{\varphi} / \kappa\right)$ is obtained from the first two equations of (11):

$$
\begin{aligned}
& \frac{1}{h} \frac{\epsilon_{x}}{\kappa}=\frac{N_{x}}{N_{0}}-\frac{N_{\varphi}}{N_{0}}+\frac{1}{2}, \\
& \frac{1}{h} \frac{\epsilon_{\varphi}}{\kappa}=-\frac{N_{x}}{N_{0}}+\frac{2 N_{\varphi}}{N_{0}}-1
\end{aligned}
$$

or

$$
N_{0} \epsilon_{x}: N_{0 \epsilon_{\varphi}}: M_{0} \kappa=4\left(\frac{N_{x}}{N_{0}}-\frac{N_{\varphi}}{N_{0}}+\frac{1}{2}\right): 4\left(-\frac{N_{x}}{N_{0}}+2 \frac{N_{\varphi}}{N_{0}}-1\right): 1 .
$$


If on the other hand the partial derivatives of $F$ of Eq. (12) are compared with (15), one obtains

$$
N_{0} \epsilon_{x}: N_{0} \epsilon_{\varphi}: M_{0} \kappa=\frac{\partial F}{\partial\left(N_{x} / N_{0}\right)}: \frac{\partial F}{\partial\left(N_{\varphi} / N_{0}\right)}: \frac{\partial F}{\partial\left(M_{x} / M_{0}\right)} .
$$

Equation (16) establishes the following important fact: if a critical combination $N_{x}, N_{\varphi}, M_{x}$ is represented by a point $H$ on the boundary of the safe domain, and a corresponding plastic deformation by the vector $\mathrm{HG}$ with the components $N_{0} \epsilon_{x}, N_{0} \epsilon_{\varphi}$ and $M_{0} \kappa$, this vector has the direction of the exterior normal to the boundary of the safe domain at the point $H$ (Fig. 6). This important result may be interpreted as a special form of Hill's principle of maximum plastic work [6].

It is easily shown that this result applies to the other parts of the yield surface. However the edges where two analytically different parts of the yield surface meet require special attention. If across such an edge the yield surface does not have a continuously turning normal, the flow mechanism corresponding to the stress-state represented by a point on this edge is not uniquely determined. It can be shown that such flow mechanisms are mapped to the domains $(C, B),(C, D),(E, F)$ and $(A, F)$ of $\epsilon_{x} / \kappa$ and $\epsilon_{\psi} / \kappa$ plane (Fig. 3) and the strain-rate vector with the components $N_{0} \epsilon_{x}, N_{0} \epsilon_{\varphi}$ and $M_{0} K$ is a linear combination (with positive coefficients) of two normals to the adjacent surfaces at the considered point.

In a corner like $B^{\prime}$, however, the strain-rate vector is a linear combination (again with positive coefficients) of the normals of three surfaces meeting in $B^{\prime}$.

4. The mathematical problem. Examples. Consider a shell of length $l$, under the internally distributed load $\lambda \cdot p(x)$ and the axial force $\lambda N$ per unit length of the shell cross-section.

The basic problem of limit analysis is to determine the value of $\lambda$ for which the plastic collapse of the shell occurs under the various end conditions.

Physical conditions which must be fulfilled when the plastic collapse occurs require the formulation of the following mathematical problem.

It is required to find the functions $N_{\varphi}(x), M_{z}(x), Q_{x}(x)$ and $u(x)$ and $w(x)$ in such a way that

(1) $M_{z}$ is continuous, with piecewise continuous first derivatives; $N_{\varphi}$ and $Q_{z}$ are piecewise continuous; $u$ and $w$ are continuous and have piecewise continuous first and second derivatives.

(2) $N_{\varphi}, M_{x}$ and $Q_{x}$ satisfy the equations of equilibrium (4), in which $N_{x}=\lambda N$ and $p$ must be replaced by $\lambda p$.

(3) The point with coordinates $N_{x} / N_{0}, N_{\varphi} / N_{0}$ and $M_{x} / M_{0}$ lies inside or on the yield surface of Fig. 6: in the first case $d w / d x$ and $d u / d x$ must vanish, in the second case the strain-rate vector $N_{0} \epsilon_{x}, N_{0} \epsilon_{\varphi}, M_{0} \kappa$ defined by the Eqs. (5), (6) and (7) must satisfy the normality condition indicated in the previous section.

(4) The end conditions which will be mentioned later must be satisfied.

In the problem to be discussed the internal load is uniformly distributed $[p(x)=c t=p]$ and the free end of the shell is carrying the axial load $N\left(-1<N / N_{0}<0\right)$ per unit length of the circumference. The other end of the shell is built-in (Fig. 7). First of all we assume that $N$ is given, and we shall determine the value of $p$ which causes collapse. This is equivalent to the solution of the basic problem. 
It is obvious from the equilibrium considerations that, when the plastic collapse occurs, the active plastic states of the shell elements are situated on the intersection of the yield surface with the plane $N_{x} / N_{0}=N / N_{0}$. Such typical intersections are shown
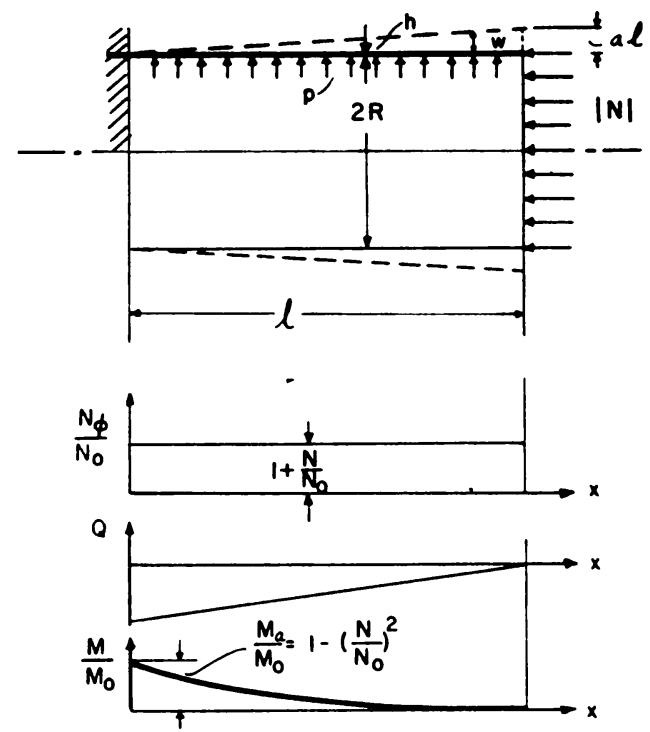

FIG. 7

in Fig. 5c. This type of yield curve is essentially a rectangle with the corners rounded off by the parabolic arcs. The sides of the rectangle are given by the following equations

where

$$
\frac{N_{a}}{N_{0}}=1+\frac{N}{N_{0}}, \quad \frac{M_{a}}{M_{0}}=1-\left(\frac{N}{N_{0}}\right)^{2} \quad \text { and } \quad \frac{N_{\varphi}}{N_{0}}=-1,
$$

$$
-1<\frac{N}{N_{0}}<0 .
$$

As a first approximation we shall neglect the parabolic corners, and consider the complete rectangle as the relevant yield curve. The flow rule corresponding to this approximate yield curve is such that the vector with the components $\kappa$ and $\epsilon \varphi$ is perpendicular to the sides of the rectangle of Fig. $5 \mathrm{~d}$. The choice of $\epsilon_{x}$ depends on the shape of the approximate convex polyhedron. We shall assume the following rule:

$\epsilon_{x}: \epsilon_{\varphi}: \kappa=-1: 1: 0$ when $N_{\varphi}=N_{a} \quad$ and $-M_{a}<M_{x}<M_{a}$,

$\epsilon_{x}: \epsilon_{\varphi}: \kappa=0:-1: 0$ when $N_{\varphi}=-N_{0}$ and $-M_{a}<M_{x}<M_{a}$.

In the corner where $N_{\varphi}=N_{a}$, and $M_{x}= \pm M_{a}$ we specify only the following:

$$
\epsilon_{x}: \epsilon_{\varphi}: \kappa=0: 0: \pm 1
$$

is a possible flow mechanism.

Now we can determine the edge conditions more precisely. At the free edge $(x=l)$

$$
M_{x}=Q_{x}=0,
$$


at the built-in edge, $w=0$ and either $d w / d x=0$ or $M_{z}= \pm M_{a}$. In the latter case the edge becomes a yield hinge [7].

For the problem under consideration let us assume that a hinge develops at the built-in edge and

$$
\left(M_{x}\right)_{x=0}=M_{a} \text {. }
$$

The equilibrium equations (4) and (20), (21) then require that the shell should be in the regime $Q R$ (Fig. 5d) and therefore

$$
\frac{N_{\varphi}}{N_{0}}=\frac{N_{a}}{N_{0}}=1+\frac{N}{N_{0}} .
$$

From (20), (21), (22) and (4) we obtain

$$
p=\frac{N_{0}}{R}\left(1+\frac{N}{N_{0}}\right)+\frac{2}{l^{2}} M_{0}\left[1-\left(\frac{N}{N_{0}}\right)^{2}\right] .
$$

The corresponding stress-resultant distributions are shown in Fig. 7.

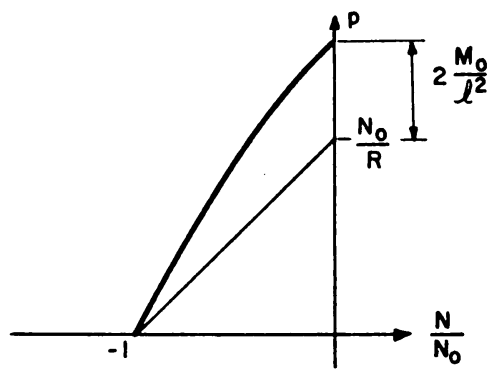

FIG. 8

Before these results can be accepted, however, it must be shown that they are associated with the velocity distributions $u(x)$ and $w(x)$ that fulfil the requirements of (18) and (19).

It is obvious that

$$
w=a x, \quad u=-\frac{a x^{2}}{2 R}
$$

are acceptable velocity distributions and the assumption of the hinge circle at the built-in edge is justified. If the second theorem of limit analysis [5] is applied using (24), one obtains (23) once again.

\section{BIBLIOGRAPHY}

1. R. Hill, On the state of stress in a plastic-rigid body at the yield point, Phil. Mag. (7) 42, 868-875 (1951)

2. D. C. Drucker, H. J. Greenberg and W. Prager, The safety factor of an elastic-plastic body in plane strain, ASME, paper No. 51-A3

3. D. C. Drucker, H. J. Greenberg, and W. Prager, Extended limit design theorems for continuous media, Quart. Appl. Math. 9, 381-389 (1952)

4. D. C. Drucker, Limit analysis of cylindrical shells under axially-symmetric loading, Proc. 1st Midwest. Conf. Solids Mech., Urbana, Ill. 1953, pp. 158-163

5. E. T. Onat and W. Prager, Limit analysis of arches, J. Mech. \& Phys. Solids, 1, 77-89 (1953)

6. R. Hill, Quart. J. Mech. Appl. Math. 1, 18 (1948)

7. H. G. Hopkins and W. Prager, The load carrying capacities of circular plates, J. Mech. \& Phys. Solids, 2, 1-13 (1953) 